\title{
Nutrient-sensing and autophagic genes in fed and fasted states
}

Two new studies published in Nature provide insights into mechanisms that underlie autophagy in the context of normal metabolic responses.

Regulation of autophagy, a catabolic process by which cells remove damaged and misfolded proteins, has largely been studied under conditions of stress and nutrient deprivation. Now, findings from two independent research groups in the USA report that autophagy is not only an acute response to cellular stress, but is a process that is integral to maintaining cellular metabolic homeostasis. "This research integrates autophagy with normal metabolic responses rather than as an emergency response to extreme starvation," says David Moore, senior investigator of one study.

Observations that the nutrientsensing nuclear receptors peroxisome proliferator-activated receptor- $\alpha$ (PPAR- $\alpha$ ) and farnesoid $\mathrm{X}$ receptor (FXR) are activated in the liver in fasted and fed states, respectively, led Moore and colleagues to hypothesize that these proteins might be involved in the transcriptional control of autophagy. In an elegant series of in vitro and in vivo experiments, they showed that protein levels of an autophagosome marker, as well as mRNA transcripts encoding autophagic genes, were upregulated in the livers of fasted and chow-fed mice. This induction of autophagy was further increased in both groups of mice following treatment

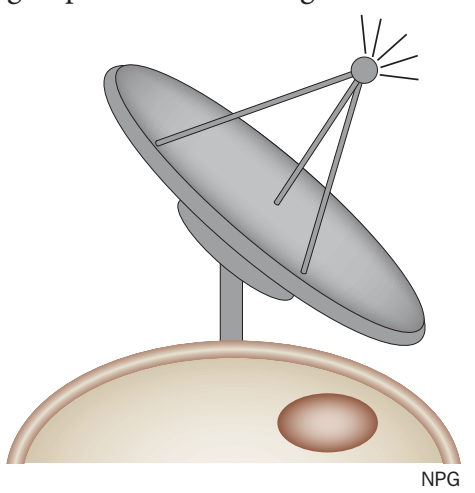

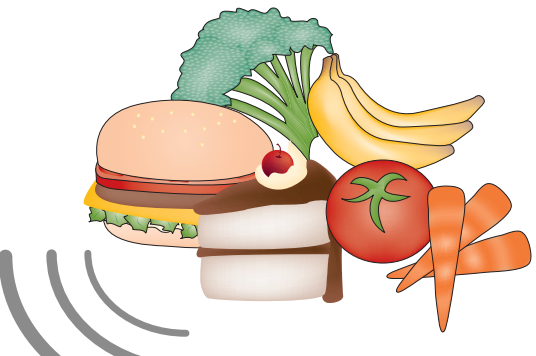

$\longrightarrow$ with a PPAR- $\alpha$ agonist and suppressed in mice treated with an FXR agonist, which suggests that these receptors have complementary functions.

Another study led by Jongsook

Kim Kemper identified an enrichment of FXR binding sites at transcription start sites of genes associated with autophagy and postulated that FXR might function to transcriptionally repress hepatic autophagy in the fed state. Interrogation of publically available data sets also demonstrated that binding of the fasting transcriptional activator cAMP response-element binding protein (CREB) was enriched upstream of genes associated with autophagy. Using biochemical analysis and experiments in a mouse hepatic cell line, they showed that CREB-mediated activation of autophagy is antagonized by FXR activity.

Investigators from both groups believe that these findings have important clinical implications. "Our discoveries reveal potential novel targets for developing new drugs for the treatment of human diseases that are associated with autophagy dysfunction, including metabolic disorders, neurodegenerative diseases and cancer," explains Kemper.

\section{Jennifer Sargent}

Original articles Lee, J. M. et al. Nutrientsensing nuclear receptors coordinate autophagy. Nature doi:10.1038/nature13961 | Seok, J. et al. Transcriptional regulation of autophagy by an FXR-CREB axis. Nature doi:10.1038/nature13949 